\title{
Filum terminale paraganglioma with associated cyst: a case report
}

\author{
Danil Adam $^{1,2}$, Cristiana Moisescu ${ }^{2}$, Dragos Iftimie ${ }^{2}$, Gina Burdusa ${ }^{2}$ \\ ${ }^{1}$ „Carol Davila” University of Medicine and Pharmacy, Bucharest, ROMANIA \\ ${ }^{2}$ Department of Neurosurgery, „Saint Pantelimon” Clinical Emergency Hospital, Bucharest, \\ ROMANIA
}

\begin{abstract}
Background and importance: Paragangliomas are neuroendocrine tumors that occur most often within the adrenal glands. The most frequent extra-adrenal locations include the carotid body and the jugular bulb. Filum terminale paragangliomas are extremely rare, with just 33 cases reported to date. Imagistic appearance is similar with other types of intradural extramedullary tumors such as ependymomas and schwannomas. Histopathological examination is the only method of establishing a definitive diagnosis. They are classified as grade I WHO tumors with favorable prognosis in the case of total resection. Clinical presentation: A 46-year-old woman without any relevant medical history was admitted to our Neurosurgical Department, complaining of low back pain with bilateral sciatica. She presented no neurological deficits and routine blood tests, as well as heart rate and blood pressure, were within normal ranges. Lumbar spine MRI with gadolinium enhancement revealed an intradural tumor with irregular, well defined margins and intense homogeneous enhancement, located at the level of the L3 vertebra. It presented an unenhancing intradural cystic lesion that extended cranially up to the level of the L1 vertebra. The tumor and associated cyst were completely resected through L2-L4 laminectomy. The tumor-cyst complex was attached, yet nonadherent to the conus medullaris and nerve roots, which allowed safe total removal. Histopathological examination was suggestive for paraganglioma, a diagnosis confirmed by immunohistochemistry that classified it as moderately differentiated, non-secreting type. Serum metanephrines subsequently determined were within normal ranges. Also, additional imagistic tests consisting of cervical and abdominal ultrasound and contrast enhanced CT scans did not detect any additional tumors. Postoperatively, the patient was neurologically intact, with complete remission of presenting symptoms at 3 months follow-up. Conclusion: Filum terminale paraganglioma is a rare diagnosis which is frequently overlooked. However, gross total resection, a common goal for all spinal tumors, can be curative in these particular cases.
\end{abstract}

Key words: paraganglioma, filum terminale, imagistic differential diagnosis 


\section{Background and importance}

Paragangliomas are neuroendocrine system tumours which regularly occur within the adrenal medulla. Most frequent extraadrenal locations are represented by carotid body, glomus jugulare, mediastinum and retroperitoneum. (1)

In extremely rare situations, paragangliomas can develop within the spinal canal, usually confined to the cauda equina, with approximately 200 such cases described in the literature. (2)

Originally described in 1970 as a "secretory ependymoma" (3) and two years later defined as a distinctive tumour by Lerman et al (4), paragangliomas present a key histological characteristic, namely the presence of cytoplasmic granules which contain catecholamines. Considering spinal paragangliomas, like most other extra-adrenal lesions, the majority are non-functional neoplasms. (5)

Spinal paragangliomas usually present with radicular pain and neurological deficits and, since cauda equina is often involved, they may cause cauda equina syndrome. Magnetic resonance imaging (MRI) represents the investigation of choice in evaluating lesions of the cauda equina. Due to their similarities in imagistic appearance, paragangliomas can be easily mistaken for ependymomas or schwannomas. (6) Spinal paragangliomas are benign lesions and they need to be distinguished from more aggressive tumour pathology, considering that their prognosis is excellent after total surgical resection, which currently represents the gold standard in treatment. (7)

We present the case of a patient with filum terminale paraganglioma, addressing the clinical and imagistic characteristics, as well as histopathological aspects.

\section{Clinical presentation}

A 46-year-old woman was admitted to our department with a one - year history of low back pain and bilateral sciatica, symptoms which have aggravated over the past two months. Her general physical examination was normal. The neurological examination revealed an anteflexion of $70^{\circ}$ and a bilateral straight leg raise test of $80^{\circ}$. Motor and sensory examination, deep tendon reflexes, peri-anal sensitivity and anal tone were within normal range. Also, the patient had no history of bowel or bladder dysfunction. Routine blood tests, as well as heart rate and blood pressure, were within normal ranges.

Due to persistent symptoms, an enhancing magnetic resonance imaging examination of the lumbar spine was performed. The study revealed an intradural mass (isointense in T1 sequence and hypointense in $\mathrm{T} 2$, respectively) located at the level of the inferior half of L3 vertebra, developed within the cauda equina as a uniformly enhancing nodule with irregular, polylobular contour. The described lesion measured $15.5 \mathrm{~mm}$ in antero-posterior diameter and occupied approximately $85 \%$ of the spinal canal. It also associated a cystic lesion that extended cranially up to the level of L1 intervertebral disc, at the top of the conus medullaris, providing an overall distended aspect of the spinal canal (Figure 1, Figure 2). 

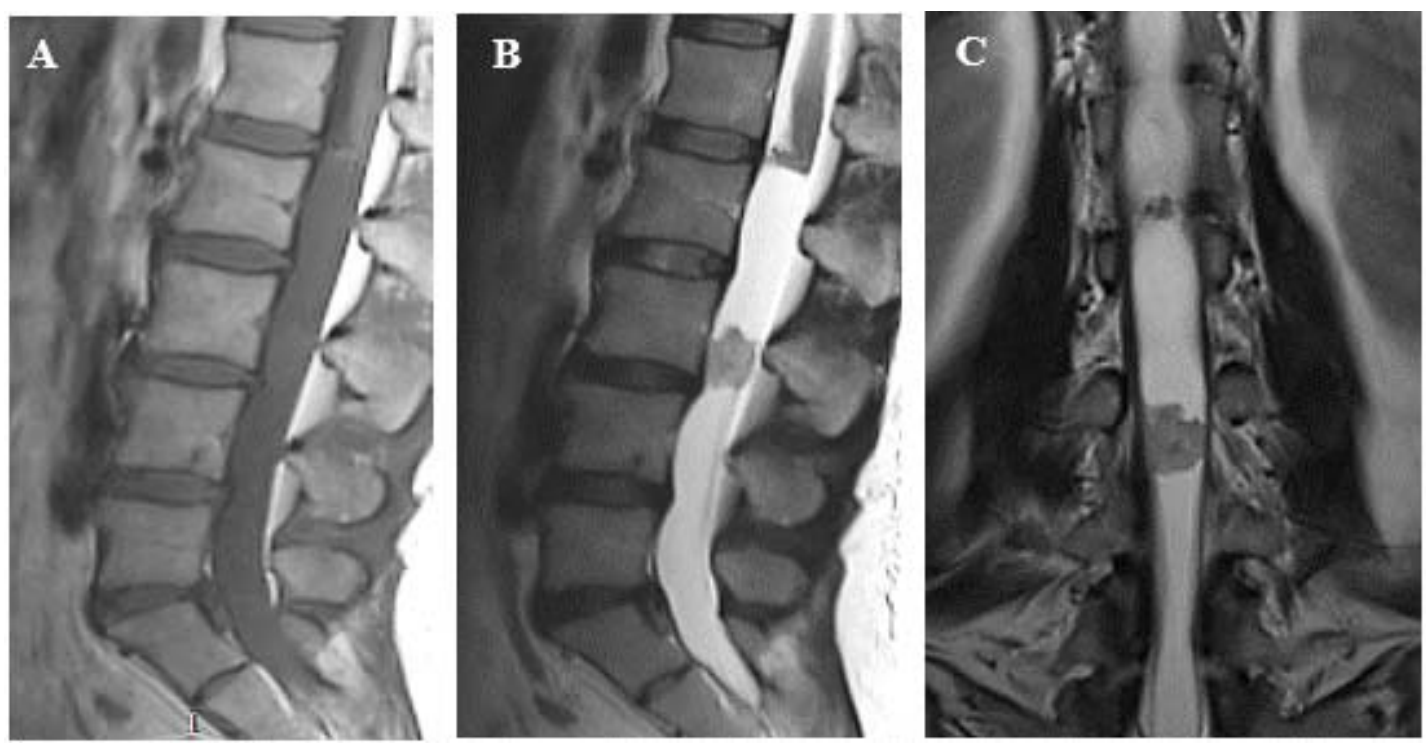

Figure 1 - Ovoidal intradural mass developed at the level of cauda equina, isointense in T1-weighted MRI images (A), hypointense in T2-weighted MRI images, in sagittal (B) and coronal (C) sections, presenting an overlying cystic lesion extended up to the level of L1 vertebra
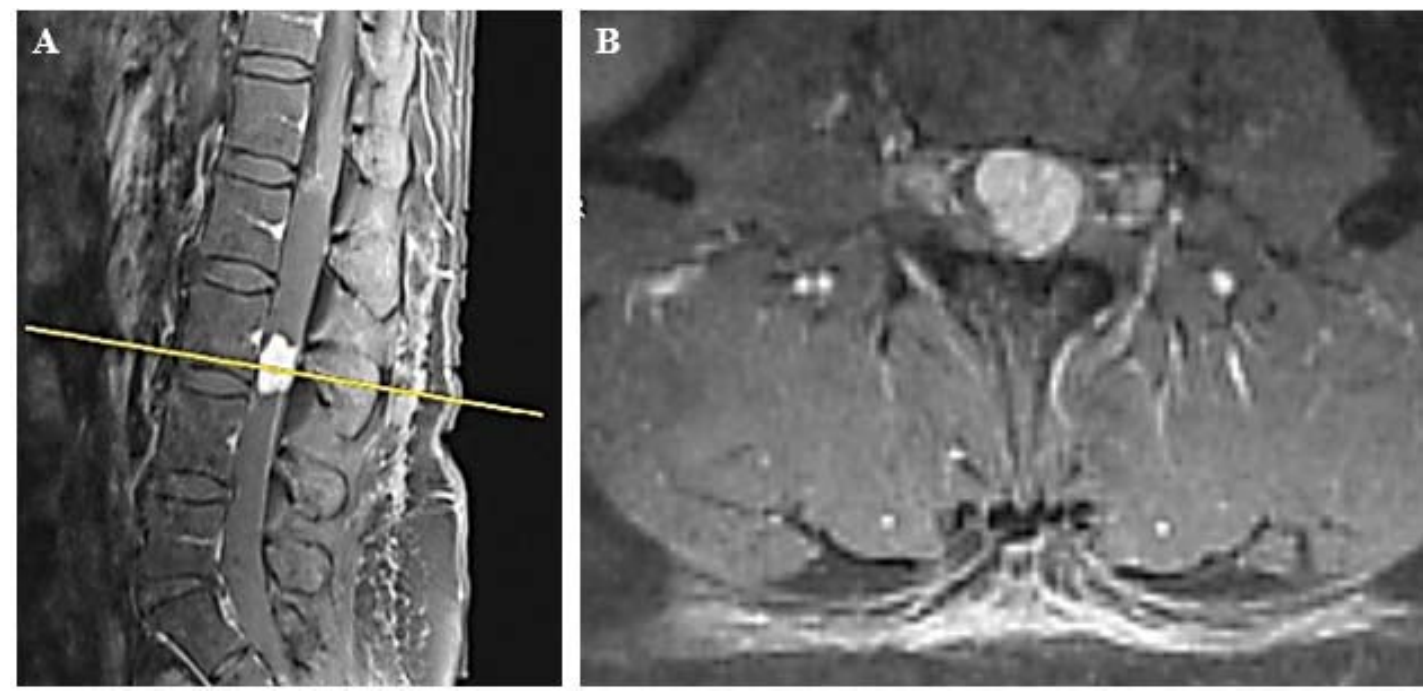

Figure 2 - The tumour showed homogenous enhancement after injection of Gadolinium in sagittal (A) and axial (B) T1 contrast - weighted MRI sequences

The patient was brought in for surgery and a L2-L4 laminectomy was performed. After opening the dura mater, an oval shaped, red- coloured, with firm consistency, vascular, well-circumscribed mass was identified (Figure 3). 


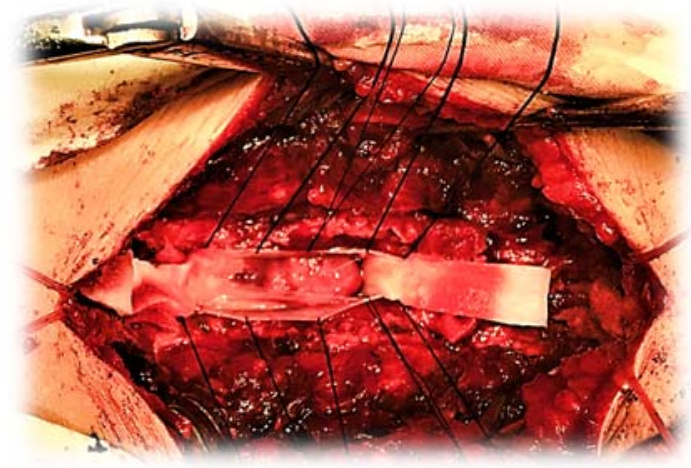

Figure 3 - Intraoperative image: after opening the dura mater, a red-coloured, firm, well-circumscribed mass contiguous with the filum terminale nerve roots was identified

Although contiguous with filum terminale nerve roots at its inferior aspect, the tumour was non-adherent to the dura mater, nor to the spinal roots. These characteristics allowed a total en bloc removal of the tumour and its cystic component (Figure 4).

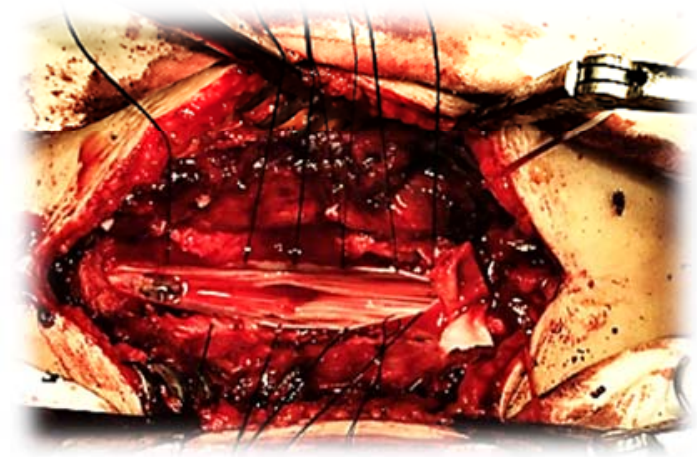

Figure 4 - Intraoperative image showing complete removal of cauda equina tumour and its cystic component

The patient presented an uneventful postoperative course. The radicular pain subsided completely and the neurological examination was normal at discharge, as well as at 3 months follow-up.

The histopathological examination of the tumour revealed small nests of chromaffin cells (chief cells) with pale eosinophilic staining separated into groups by segmenting bands of fibrovascular stroma, surrounded by supporting sustentacular cells, aspect known as Zellballen pattern (Figure 5). Immunohistochemical testing showed a positive reaction for chromogranin and a negative reaction for glial fibrillary acidic protein (GFAP) in the chief cells. All these findings supported the diagnosis of paraganglioma. It was further classified as moderately differentiated, non-secreting type.

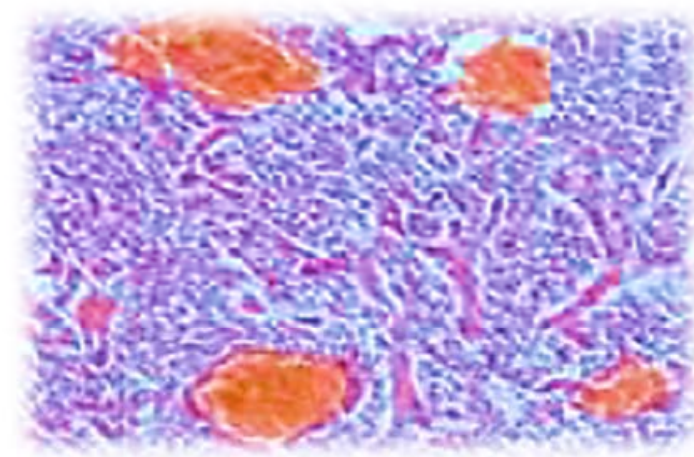

Figure 5 - Histopathological examination of the tumour showing typical Zellballen architecture H\&E (x 10)

Taking into consideration the diagnosis, further investigations were conducted, in order to identify secondary paragangliomas, if present. Abdominal ultrasonography, cervical spine and abdominal contrast enhanced CT scan and cervical Doppler were performed, that did not find any other tumors. Serum metanefrines were also within normal ranges. 


\section{Discussion}

The majority of paragangliomas - over $90 \%$ of cases - develop in the adrenal gland whereas approximately $90 \%$ of extra-adrenal paragangliomas occur in the carotid body and jugular gland. (8) The development of paragangliomas in the central nervous system is extremely rare. Intracranially, they have been described in the sellar, pineal and petrous ridge region. (9) Regarding the spinal canal, around 200 paragangliomas with this location have been described so far, mostly intradural. In particular, only 33 cases of filum terminale paraganglioma have been reported in the literature. (10) They can also develop extradurally but most extradural spinal paragangliomas are metastatic tumours, appearing more frequently at the level of the thoracic spine. $(11,12)$ The case that we reported was a spinal paraganglioma located at the level of the filum terminale, representing a solitary case among the spinal tumours diagnosed in our department over the last two years.

The clinical presentation usually depends on the location and size of the tumour, radicular pain and neurological deficits being most frequently encountered. However, paragangliomas can occasionally cause uncommon manifestations such as accelerated heart rate and arterial hypertension due to overproduction of catecholamines (2), manifestations which we did not encounter in our case.

The gold standard imagistic investigation for spinal pathology is MRI, however filum terminale paragangliomas do not present specific MRI characteristics. Herman et al. described in an article published in 1998 the MRI characteristics of spinal paragangliomas and reported that MRI fails in differentiating paragangliomas from other tumours. (13) Paragangliomas present as hypo- or isointense masses in $\mathrm{T} 1$ - weighted images, hypointense in T2 - weighted images, with diffuse contrast enhancement. Nevertheless, other spinal tumours, such as ependymomas, have similar imagistic appearance which makes the preoperative radiological diagnosis of paraganglioma extremely difficult.

In our case, the tumour had no particular MRI features: it presented as an ovoidal intradural mass developed at the level of cauda equina, isointense in T1-weighted images, hypointense in $\mathrm{T} 2$-weighted images, with homogenously contrast enhancement. The mass presented an associated cystic lesion extending cranially to the level of L1 vertebra with complex signal intensity (Figure 1, Figure 2). The pathogenesis of the cyst formation is not completely understood but it is not an unusual occurrence in association with paragangliomas. In 1997, Faro et al reported a case of paraganglioma of the cauda equina with an associated overlying intramedullary cyst. The author offered two possible hypothesis for cyst development: firstly, the normal flow of CSF could have been obstructed inferiorly by the tumour thus creating a differential pressure, forcing fluid into the spinal cord; secondly, the cysts could have been created by the fluid from abnormal tumour vessels extravasated into the interstitial space in the spinal cord. (9) Nevertheless, the presence of overlying cystic 
lesions is not characteristic for paragangliomas, as they occur in association with a considerable number of spinal tumours, including ependymomas.

Microscopically, paragangliomas show typical nuclear monomorphism and display "Zellballen" pattern (large chief cells arranged in nests). Immunohistochemistry studies show reactivity for markers of neural differentiation, including chromogranin and synaptophysin and lack of reactivity for glial fibrillary acidic protein (GFAP). This particular immunoprofile, which we encountered in our case as well, is helpful in order to exclude ependymoma from the differential diagnosis, considering that ependymomas are immunoreactive for GFAP and S-100 protein.

The recommended treatment for spinal paragangliomas is total resection. In such cases, the postoperative prognosis is very good, associating a recurrence rate of only $4 \%$ at 12 years after surgery. (15) In case of subtotal resection, radiotherapy may be used, although resistance to this type of treatment has been noted. Chemotherapy has no therapeutic role. (16)

Few cases of metastatic paraganglioma have been reported in the published literature. $(11,12)$ The patient that we presented was further investigated in order to identify the potential presence of other tumors. However, there was no evidence of secondary paragangliomas.

\section{Conclusion}

Filum terminale paragangliomas are benign tumours which, in the majority of cases, can be successfully treated by total resection. Although extremely rare, they should be included in the differential diagnosis of spinal intradural tumours as they need to be distinguished from more aggressive tumours, given the excellent prognosis that they associate.

\section{References}

1. Lack EE. Paragangliomas. In: Mills SE, ed. Sternberg's Diagnostic Surgical Pathology. Lippincott Williams \& Wilkins; 2004:669-696.

2. Gelabert-González M. Paragangliomas of the lumbar region. J Neurosurg Spine. 2005;2(3):354-365. doi:10.3171/spi.2005.2.3.0354

3. Miller CA, Torack RM. Secretory ependymoma of the filum terminale. Acta Neuropathol. 1970;15(3):240-250. http://www.ncbi.nlm.nih.gov/pubmed/4193811.

Accessed May 29, 2018.

4. Lerman RI, Kaplan ES, Daman L. Ganglioneuromaparaganglioma of the intradural filum terminale. J Neurosurg. 1972;36(5):652-658. doi:10.3171/jns.1972.36.5.0652

5. Glenner GG, Grimley PM, (U.S.) AFI of P. Tumors of the extra-adrenal paraganglion system (including chemoreceptors). 1974:90 pages. file://catalog.hathitrust.org/Record/007401029.

6. Aghakhani N, George B, Parker F. Paraganglioma of the cauda equina region--report of two cases and review of the literature. Acta Neurochir (Wien). 1999;141(1):8187. http://www.ncbi.nlm.nih.gov/pubmed/10071690. Accessed May 29, 2018.

7. Soni T V, Gupta A. Spinal Paraganglioma: A Rare Tumour.

http://medind.nic.in/gaa/t14/i1/gaat14ilp110.pdf.

Accessed May 29, 2018.

8. Pacak K, Linehan WM, Eisenhofer G, Walther MM, Goldstein DS. Recent advances in genetics, diagnosis, localization, and treatment of pheochromocytoma. Ann Intern Med. 2001;134(4):315-329. http://www.ncbi.nlm.nih.gov/pubmed/11182843.

Accessed May 29, 2018.

9. Faro SH, Turtz AR, Koenigsberg RA, Mohamed FB, Chen CY, Stein H. Paraganglioma of the cauda equina with associated intramedullary cyst: MR findings. AJNR 
Am J Neuroradiol. 1997;18(8):1588-1590. http://www.ncbi.nlm.nih.gov/pubmed/9296205.

Accessed May 29, 2018.

10. Landi A, Tarantino R, Marotta N, et al. Paraganglioma of the filum terminale: case report. World J Surg Oncol. 2009;7:95. doi:10.1186/1477-7819-7-95

11. Lau D, La Marca F, Camelo-Piragua S, Park P. Metastatic paraganglioma of the spine: Case report and review of the literature. Clin Neurol Neurosurg. 2013;115(9):1571-1574.

doi:10.1016/j.clineuro.2013.01.006

12. Lázaro B, Klemz M, Flores MS, Landeiro JA. Malignant paraganglioma with vertebral metastasis: case report. Arq Neuropsiquiatr. 2003;61(2B):463-467. doi:10.1590/S0004-282X2003000300026

13. Herman M, Pozzi-Mucelli RS, Skrap M. Paraganglioma of the cauda equina: case report and review of the MRI features. Acta Univ Palacki Olomuc
$\mathrm{Fac}$

Med.

$1998 ; 141: 27-30$

http://www.ncbi.nlm.nih.gov/pubmed/9684477.

Accessed May 29, 2018.

14. Suri VS, Tatke M, Singh D, Sharma A. Histological spectrum of ependymomas and correlation of p53 and Ki67 expression with ependymoma grade and subtype. Indian J Cancer. 41(2):66-71. http://www.ncbi.nlm.nih.gov/pubmed/15318011.

Accessed May 29, 2018.

15. Makhdoomi R, Nayil K, Santosh V. Primary Spinal Paragangliomas: A Review. Neurosurg Q. 2009;19(3):196-199. doi:10.1097/wnq.0b013e3181a45c15 16. Say CC, Hori J, Spratt J. Chemodectoma with distant metastasis: case report and review of the literature. Am Surg. 1973;39(6):333-341 http://www.ncbi.nlm.nih.gov/pubmed/4350420. Accessed May 29, 2018. 